\title{
Analysis of Collision Probability in Unsaturated Situation
}

\author{
Qian Dong \\ Chair of Computer Networks \\ Faculty of Computer Science \\ Technical University of Dresden \\ 01062 Dresden \\ s7686783@mail.inf.tu-dresden.de
}

\author{
Waltenegus Dargie \\ Chair of Computer Networks \\ Faculty of Computer Science \\ Technical University of Dresden \\ 01062 Dresden \\ waltenegus.dargie@tu-dresden.de
}

\begin{abstract}
A large number of the MAC protocols proposed for establishing wireless sensor networks are based on the 802.11 standard. The trade-off in these protocols is the control packet overhead and the retransmission cost due to collision without it. In this paper we evaluate the collision probability of a CSMA/CA MAC protocol in an unsaturated situation as a function of nodes' sampling and transmission rates. We provide an accurate and comprehensive analytical model in which a finite number of nodes exist. We assume an ideal channel condition, independent collision probability of packets as well as infrequent communication between sensor nodes. We will demonstrate that the collision probability changes from 0 to 0.22 as the sampling rate changes from 0 to $0.94 \mathrm{Mbps}$. Moreover, we will demonstrate that collision only begins after the sampling rate reaches $0.31 \mathrm{Mbps}$, which implies that for a sampling rate below this threshold, the control overhead can be avoided by altogether avoiding the collision avoidance mechanism.
\end{abstract}

\section{Categories and Subject Descriptors}

C.2.1 [Computer-Communication Networks]:

Network Architecture and Design[Wireless communication]; C.4 [Performance of Systems]: Modeling techniques

\section{General Terms}

Performance, design, measurement

\section{Keywords}

Wireless sensor networks, collision, unsaturated situation, medium access control, energy-consumption

\section{INTRODUCTION}

Wireless sensor networks have a large number of applications [4], [7]. These networks normally consist of a large number of nodes with sensing, computation, communication and in-network processing capabilities. Each sensor

Permission to make digital or hard copies of all or part of this work for personal or classroom use is granted without fee provided that copies are not made or distributed for profit or commercial advantage and that copies bear this notice and the full citation on the first page. To copy otherwise, to republish, to post on servers or to redistribute to lists, requires prior specific permission and/or a fee.

SAC'10 March 22-26, 2010, Sierre, Switzerland.

Copyright 2010 ACM 978-1-60558-638-0/10/03 ...\$10.00. node has one or more sensors which sample raw data in their sensing filed. The embedded low-power radios enable a wireless communication between neighboring nodes. Since sensor nodes are battery operated, the energy consumption of MAC protocols is an essential design issue. In this paper, the performance of the collision probability of a contentionbased protocol in an unsaturated situation will be evaluated. We provide an accurate mathematical model to quantify the collision probability of the protocol by varying the sampling rate.

The rest of this paper is organized as follows: in section 2, we introduce related work. In section 3 , we provide a detailed account of the network model and its basic parameters as well as the relationship between the parameters. Moreover, a theoretical mathematical model is developed which will be the basis for our simulation. In section 4 and 5 the behavior of the collision probability in the unsaturated situation is visualized using simulation and the observation is interpreted. Finally, in section 6 , we give a concluding remark.

\section{RELATED WORK}

So far, there are several solutions addressing the problem of energy waste in wireless sensor networks. Collision probability as one of the major causes of energy consumption has been investigated extensively [1], [2]. In [1], Bianchi uses the Markov Chain model to calculate the collision probability in the saturated situation in order to estimate the 802.11 DCF throughput. The impact of channel capture following a busy period on collision probabilities in the saturated 802.11 network is studied in [6]. These approaches mainly focus on the saturated situation with the assumption that the sampling rate is not less than the transmission rate, which is however not the case in many wireless sensor networks.

Packet collision significantly affects the performance as well as the energy consumption of wireless sensor networks. Collision itself is affected by several factors, such as the average number of contending neighbors and the sampling (sensing) rate. For the analysis model we presented here, a detailed description of the deployment setting is given elsewhere ( [5], [4], and [3]). The setting assumes the presence of $N$ sensor nodes that are uniformly distributed in a rectangular area with the size of $(a \times b)$. The density of the network is expressed as, $\lambda=\frac{N}{a \times b}$. By considering the radio transmission range, $R$, the average number of nodes within a radio transmission area is: $N_{\text {avg }}=\left\lfloor\frac{N}{a \times b} \times \pi R^{2}\right\rfloor$. The symbol \lfloor\rfloor denotes the floor integer value, since the average number of nodes should never be decimal. 


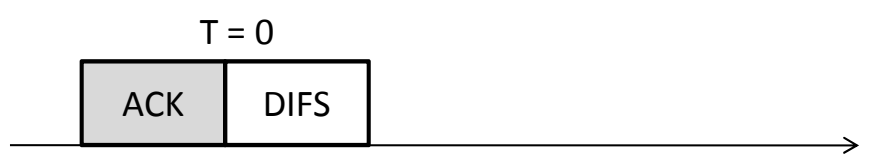

(a)

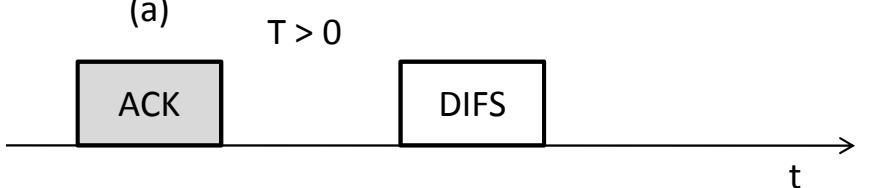

(b)

Figure 1: The saturated and unsaturated situation with regard to $T$

\section{COLLISION PROBABILITY MODEL}

Suppose there are $N$ nodes in an area of $a \times b$, where $a \times b$ is the area of deployment and $a \leq b$. If one of the nodes is assumed to be located in the middle of the radio transmission area, the average number of neighbors for a given transmitter or receiver can be expressed as:

$$
N_{\text {neighbor }}=\left\lfloor\frac{N}{a b} \times \pi R^{2}\right\rfloor-1
$$

The collision probability during the medium access depends on how often the nodes communicate with each other. We can define two scenarios: The first one is the saturated situation, in which the nodes always have packets to send. This assumes that the sampling rate is close to or larger than the transmission rate. However, the saturated case occurs rarely in real sensor network applications. The second one is the unsaturated situation, in which the sampling rate is much less than the transmission rate. This is a more accurate description of real networks. Figure 1 illustrates these two scenarios.

In a contention based MAC protocol based on the IEEE 802.11 standard, after the successful transmission of the $A C K$ frame by the receiver, the channel becomes free and a new round of competition starts. Therefore, all the neighbor nodes of the transmitter and the receiver will finish waiting and begin to contend for the medium. If these neighbors have some available sampled packets to transmit, they will contend for the medium immediately. Contention is made by monitoring the channel for a duration called the distributed inter-frame space, DIFS. In the saturated situation, the DIFS interval immediately follows the previous ACK frame. As a result, the time interval T (see figure 1(a)) between the end of the previous ACK frame and the beginning of the new DIFS sensing period equals to zero. In the unsaturated situation, however, after the ACK frame is successfully transmitted, since the newly sampled data packet has not arrived in the queue yet, the neighbor nodes cannot contend for the medium at that moment. Therefore, the time interval $\mathrm{T}$ (see figure $1(\mathrm{~b})$ ) is larger than zero. The fundamental reason for the difference between the saturated and unsaturated situation is the relationship between the sampling rate and the transmission rate. In the former case, the sampling rate is close to or larger than the transmission rate; in the latter case, however, the sampling rate is much smaller than the transmission rate.

\subsection{Backoff Model}

As soon as a node senses the channel idle for a period of DIFS, its backoff timer starts to decrease. Only when the timer reaches zero can a node begin its RTS transmission. When the backoff is initiated, a random integer backoff time is selected within the range $\left(0,2^{i} C W_{\min }-1\right)$, where the first contention window is $\left(2^{0} C W_{\min }-1\right)$. So the average backoff time in the first transmission attempt is $\frac{\left(2^{0} C W_{\min }-1\right)}{2}$. If $p$ presents the collision probability in the unsaturated situation, then the probability that the RTS frame is successfully transmitted in the first transmission attempt is $(1-p)$. However, if the first transmission fails, the probability of the successful transmission of the RTS frame in the second attempt is $p(1-p)$. The average backoff time in this case is $\frac{\left({ }^{1} C W_{\min }-1\right)}{2}$. The retransmission continues until the last $\left(k_{t h}\right)$ permitted transmission attempt is reached. However, when the maximum contention window arrives, the contention window will not increase anymore and thus keeps to $\left(2^{m} C W_{\min }-1\right)$. As a result, the average backoff time is kept the same as $\frac{\left(2^{m} C W_{\min }-1\right)}{2}$ from then on. In other words, from the moment that the maximum backoff stage $m$ is reached, the value of the contention window will not increase anymore though the number of the retransmission still increases until it reaches $k-1$. Thus, after transformation and simplification, the overall average backoff time can be expressed as:

$$
T_{a v g}=\left\lfloor\frac{(1-p)(A+B-C)}{1-p^{k}}\right\rfloor
$$

where:

$$
\begin{gathered}
A=\frac{C W_{\min }\left(1-(2 p)^{(m+1)}\right)}{2(1-2 p)} \\
B=\frac{p^{(m+1)} 2^{m} C W_{\min }\left(1-p^{(k-m-1)}\right)}{2(1-p)} \\
C=\frac{\left(1-p^{k}\right)}{2(1-2 p)}
\end{gathered}
$$

Equation 2 is valid on the condition that $p \in((0,0.5) \cup$ $(0.5,1))$. For $p=0.5, T_{a v g}$ has a different expression, which, however, proved to make no sense. From the equations, we can find out that the collision probability in the unsaturated situation is a function of the overall average backoff time. However, since $T_{a v g}$ is also an unknown parameter, we must look for another formula which gives us the relationship between $T_{a v g}$ and $p$.

\subsection{Collision Probability}

In the saturated situation, every sensor node always has packets to send. So for a particular node A, all its neighbors are active nodes, meaning, if one of A's neighbors, say B, is transmitting a RTS frame to A, all the RTS frames transmitted by all the other neighbor nodes of A (node C, D and $\mathrm{E}$ in the figure 2(a)) may have collision with B's transmission. The blue circles represent those active neighbors. In the unsaturated situation, however, due to the low sampling rate, not all of the neighbor nodes of $\mathrm{A}$ are active with respect to B; some of them are non-active. For example, in figure 2(b), only the RTS frames sent by $\mathrm{C}$ and D may have 


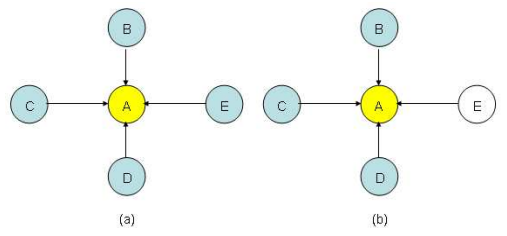

Figure 2: Active transmitting neighbors in the saturated and unsaturated situations

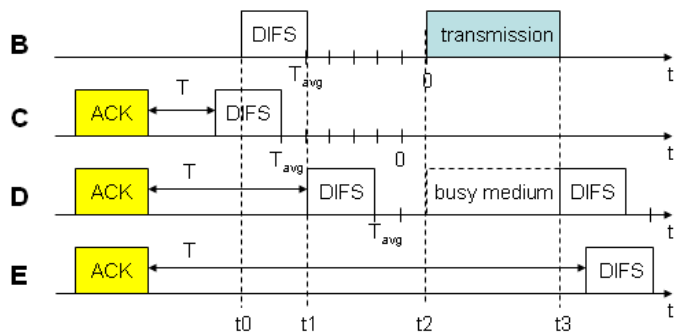

Figure 3: Three different transmission patterns according to $\mathrm{B}$

collision with the RTS frame of B, while the RTS frame E sends will never have collision with B's.

If there were $W$ distinct time slots available for contention, the probability that a node can successfully transmit a RTS packet is given as:

$$
p=\frac{M}{N_{\text {neighbor }}}\left(1-\left(1-\frac{1}{W}\right)^{M-1}\right)
$$

Since $W$ represents the average number of time slots and nodes are only permitted to transmit packets at the beginning of each time slot, $W=T_{a v g}$. Therefore, in the subsequent expressions related to equation $6, W$ is replaced by $T_{\text {avg }}$.

If we can successfully resolve equations 2 and 6 , the collision probability in the unsaturated situation can be theoretically evaluated. In this equation set, all the variables except $p$ and $T_{a v g}$ can be known by assigning a value.

\subsection{Active Neighbor Model}

We assume that all the neighbors B, C, D and E constantly transmit sampled data to A. if B's DIFS sensing time has been predetermined, there are only three different transmission patterns in the unsaturated situation of the 802.11 protocol. The difference among these three transmission patterns is the relative sensing time of the DIFS period with respect to B's DIFS time.

Nevertheless, these three patterns share some common characters, i.e., all their ACK frames occur before B's DIFS sensing period. In figure 3 , we display these three possible transmission patterns and their relationship. We assume that A's remaining neighbor nodes C, D and E represent each of these three transmission patterns, respectively. However, in reality, A does not merely have four neighbors. Hence, each node C, D and E represents multiple nodes rather than just one single node, which share one and the same transmission pattern. Unlike the others, B is a single node, for its transmission pattern has been predetermined.

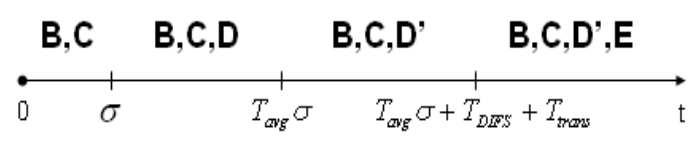

Figure 4: The Classification criterion according to $\mathbf{T}$

For all the neighbors of A, after they sense idle for a period of DIFS, they will start their randomly chosen backoff time respectively. Therefore, the backoff time for each of them is mostly different. For simplicity and efficiency reasons, we regard the different backoff times as having the same value and it is equal to the overall average backoff time, $T_{a v g}$. Since B's DIFS is predetermined, any other neighbor node of A which is contending for the medium with B must finish its packet transmission before B's DIFS. Otherwise, if the competing neighbor's ACK frame is transmitted after B's DIFS, such DIFS would not be sensed idle and thus the assumption of the validness of B's DIFS fails. Consequently, for any remaining neighbor node of $\mathrm{A}$, it must finish transmitting the ACK frame before B's idle DIFS sensing period, that is, the transmission of the ACK frame must be finished before the time t0 as showed in the figure 3.

For the remaining neighbors of A, we should also consider the possible sensing time of the DIFS. Actually, the DIFS period is allowed to occur either before $t 1$, between $t 1$ and t2 or after $\mathrm{t} 3$. In node C's transmission pattern, the DIFS occurs either before B's DIFS or simultaneously with B's DIFS. In the former case, the backoff of $\mathrm{C}$ will first decrease to zero. Thus $\mathrm{C}$ wins the channel and will transmit its RTS frame immediately afterwards. As a result, the backoff of $\mathrm{B}$ will be paused until the transmission of $\mathrm{C}$ completes. In the latter case, collision will occur since B and C simultaneously finish their backoffs and begin to transmit at the same moment. In node D's transmission pattern, the finish time of the DIFS is between $\mathrm{t} 1$ and $\mathrm{t} 2$, so the backoff of $\mathrm{B}$ will first reaches zero. In this case, the backoff time of $\mathrm{D}$ will be paused in midway as B is transmitting. In E's transmission pattern, however, the valid DIFS will only appear after the transmission of $\mathrm{B}$ at $\mathrm{t} 3$. Note that the DIFS can never be sensed idle between $\mathrm{t} 2$ and $\mathrm{t} 3$ due to the busy medium caused by the transmission of $\mathrm{B}$. Therefore, depending on these three possible time positions of the DIFS period, we classify the remaining neighbors of A into three categories, each of which matches one unique transmission pattern.

In the unsaturated situation of the 802.11 protocol, each of the remaining neighbors of A has one of those three transmission patterns according to the predetermined transmission pattern of B. However, not all of these remaining neighbors are active. Some of them are active while some of them are non-active. Since collision may only occur in C's transmission pattern, besides the node B, only those C-like remaining neighbors are active transmitting nodes. As a result, those neighbors which have the same transmission pattern as D and E have are non-active, since their transmission will never suffer collision with B's transmission.

In each of the three transmission patterns, there are several possible transmission cases. This is because those three different transmission patterns are classified by the time range of the DIFS rather than the exact time point. Therefore, in order to acquire the accurate number of the active neighbors, we need to know the ratio of the possible cases 
in the transmission pattern of those active neighbors to the total possible cases in the transmission pattern of all the neighbors.

It is important to mention that the number of possible cases in D and E's transmission pattern is mostly T related. And this number varies under different conditions. Therefore, we should evaluate the active transmitting neighbors in each condition respectively. As showed in figure 4, the time coordinate is divided into four zones and only the corresponding specific transmission patterns make sense. The notation $\sigma$ stands for the slot time, $\sigma T_{a v g}$ is overall average backoff duration, $T_{D I F S}$ represents the time period of the DIFS and $T_{\text {trans }}$ is the time of a round of transmission, which includes the time spent on the transmission of a RTS frame, a CTS frame, a data packet, an ACK frame and three SIFS phases in between. However, the retransmission time is not involved in $T_{\text {trans }}$, since another RTS frame must be transmitted to newly contend for the medium if the first transmission attempt fails. As we can observe, the transmission pattern of B certainly exists, and so does the transmission pattern of $\mathrm{C}$ no matter which time zone $\mathrm{T}$ belongs to. Note that $\mathrm{T}$ must be larger than 0 , otherwise, it will become the saturated situation. For C's transmission pattern, the number of transmission cases is a constant independent of T. On the contrary, D's transmission pattern only makes sense when $\mathrm{T}$ is equal to or larger than $\sigma$, and the number of the transmission cases dose not remain the same when $\mathrm{T}$ changes from the time interval $\left[\sigma, \sigma T_{\text {avg }}\right)$ to $\left[T_{a v g} \sigma, T_{a v g} \sigma+T_{D I F S}+T_{\text {trans }}\right)$. That's why we use the letter $\mathrm{D}$ and $\mathrm{D}$ ' in these two different time areas respectively. As to E's transmission pattern, it's only possible to appear as long as $\mathrm{T}$ is equal to or larger than $T_{a v g} \sigma+T_{D I F S}+T_{\text {trans }}$. In the following part, we will concentrate on calculating the number of the active transmitting neighbors in the unsaturated situation of the 802.11 protocol based on the classification of $\mathrm{T}$.

\subsection{1 $T \in(0, \sigma)$}

Under this condition, only the transmission pattern of $\mathrm{B}$ and $\mathrm{C}$ exists. As we analyzed before, besides the active neighbor node $\mathrm{B}$, only those $\mathrm{C}$-like neighbors are active. Obviously, here is only one possible transmission case in B's transmission pattern $\left(N_{B}=1\right)$, since it has been assumed as a point of reference to analyze all the other possible transmission patterns. However, as descried in the figure 5, there are $\left(T_{a v g}+1\right)$ possible transmission cases in C's transmission pattern which share an identical probability of occurrence.

Since only the transmission pattern of the active neighbors appears in this condition, the numerator and the denominator of the fraction which evaluates the active transmitting nodes share the same value,

$$
M=N_{\text {neighbor }}
$$

\subsubsection{T $T \in\left[\sigma, T_{\text {avg }} \sigma\right)$}

In this condition, the transmission cases in D's transmission pattern are $\mathrm{T}$ dependent. As can be seen from figure 6 , in the $D_{\frac{T}{\sigma}}{ }^{\text {th }}$ case, the finish time point of the DIFS can't reach the start time of B's transmission since $\mathrm{T}$ is smaller than $T_{a v g} \sigma$. Therefore, with the limitation of $\mathrm{T}$, there are merely $\left[\frac{T}{\sigma}\right]$ cases in all in D's transmission pattern. Hence, $N_{D}=\left\lfloor\frac{T}{\sigma}\right\rfloor$.

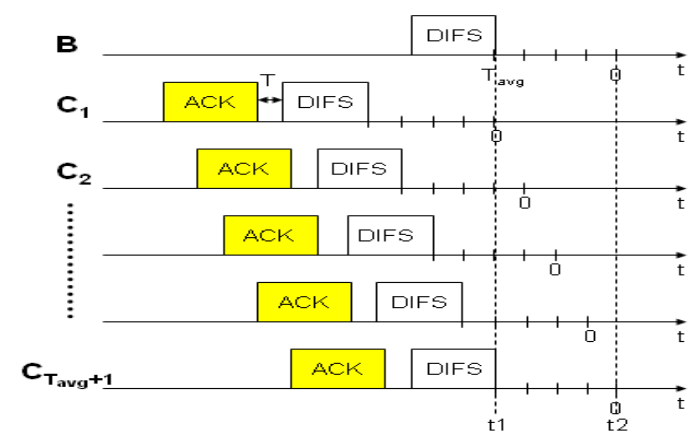

Figure 5: The transmission cases in the transmission pattern of $\mathrm{C}$

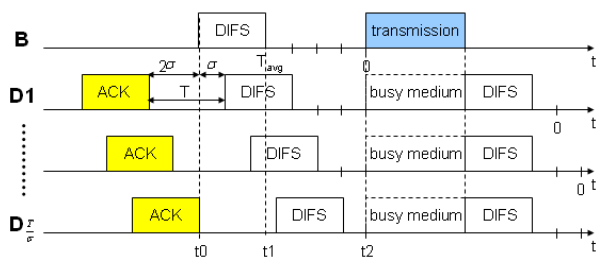

Figure 6: The transmission cases in the transmission pattern of $D$

Here, both C and D's transmission pattern exist besides B's transmission, among which, only B and C are the active transmitting neighbor nodes. Therefore, the number of the average transmitting neighbors should be evaluated as:

$$
M=\left\lfloor\frac{T_{a v g}+2}{T_{a v g}+2+\left\lfloor\frac{T}{\sigma}\right\rfloor} N_{\text {neighbor }}\right\rfloor
$$

$$
\text { 3.3.3 } T \in\left[T_{\text {avg }} \sigma, T_{\text {avg }} \sigma+T_{\text {DIFS }}+T_{\text {trans }}\right)
$$

When $\mathrm{T}$ belongs to the fourth time zone, all the transmission patterns of B, C and D could happen. However, the number of the transmission cases in D's transmission pattern changes to $T_{\text {avg }}$, since $\mathrm{T}$ is big enough now that it will not be a limitation anymore for the total number of the transmission cases. Specifically speaking, from the case in which its DIFS is closest to B's DIFS to the case in which the finish time point of its DIFS reaches B's zero backoff time point are all included, as figure 7 shows. Thus, $N_{D}=T_{\text {avg }}$.

Then the number of the active transmitting neighbor nodes in this condition should be computed as:

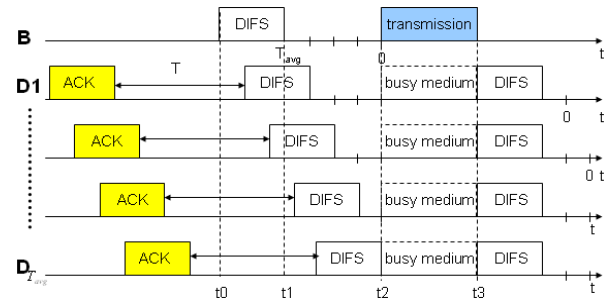

Figure 7: The transmission cases in the transmission pattern of $D$ ' 


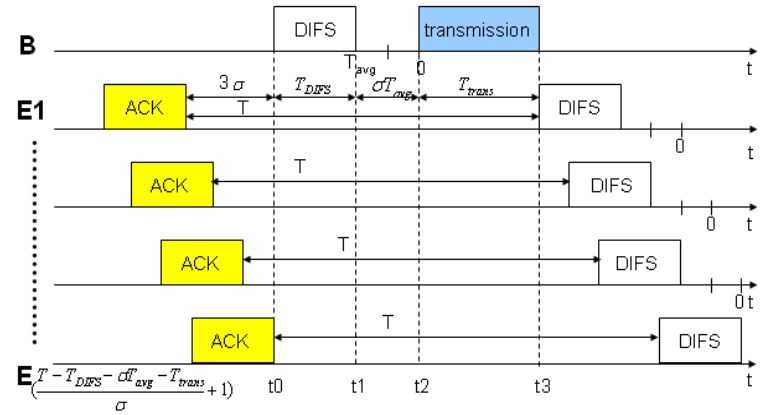

Figure 8: The transmission cases in the transmission pattern of $\mathrm{E}$

$$
M=\left\lfloor\frac{T_{a v g}+2}{2 T_{\text {avg }}+2} N_{\text {neighbor }}\right\rfloor
$$

\subsection{4 $T \in\left[T_{D I F S}+\sigma T_{a v g}+T_{\text {tran }},+\infty\right)$}

In this condition, all the transmission patterns of $\mathrm{B}, \mathrm{C}, \mathrm{D}$ and $\mathrm{E}$ make sense. There are $T_{a v g}$ cases in D's transmission pattern since $T_{a v g}$ is the maximum number of transmission cases that may take place when $\mathrm{T}$ is larger than $\sigma T_{a v g}$.

As can be observed from the figure 8, the number of the transmission cases under E's transmission pattern is:

$$
N_{E}=\left\lfloor\frac{T-T_{D I F S}-\sigma T_{a v g}-T_{\text {trans }}}{\sigma}\right\rfloor+1
$$

Here, the transmission duration $T_{\text {trans }}$ is evaluated by considering the time used for the transmission of the data packet and control packet, plus the three SIFS intervals standing between every two neighbor packets:

$$
T_{\text {trans }}=\frac{N_{R T S}+N_{C T S}+N_{D A T A}+N_{A C K}}{R_{t}}+3 T_{S I F S}
$$

After calculation and simplification, the number of active transmitting neighbors in this condition is:

$$
M=\left\lfloor\frac{T_{\text {avg }}+2}{2 T_{\text {avg }}+3+\left[\frac{T-T_{D I F S}-\sigma T_{a v g}-T_{\text {trans }}}{\sigma}\right]} N_{\text {neighbor }}\right\rfloor
$$

Apparently, the average number of active neighbors, $M$, in the unsaturated situation of the 802.11 protocol is a function of the parameters $T_{a v g}, T, \sigma, T_{D I F S}, N_{R T S}, N_{C T S}, N_{D A T A}$, $N_{A C K}, R_{t}$ and $T_{S I F S}$. All these parameters are simple except for the unknown variables $T$ and $T_{\text {avg }}$. In order to fully resolve $M$ in these different conditions, we must look for the exact value of $T$ by establishing the specific $T$ model.

\subsection{T Model}

Intuitively, the reason for the appearance of a blank time interval $T,(T>0)$ after a round of transmission in the unsaturated situation (described in the figure 9) is that the sampling rate is smaller than the transmission rate. The interval T1 is related with the transmission rate, $R_{t}$, which equals to the summation of the DIFS sensing period, $T_{D I F S}$, the overall average backoff duration, $T_{a v g} \sigma$, and the transmission time, $T_{\text {trans }}$.
Table 1: List of simulation parameters

\begin{tabular}{l|l}
\hline Basic Parameter & Default Value \\
\hline Control packet RTS/CTS/ACK & 10 bytes \\
Data message & 136 bytes \\
Transmission rate & $2 \mathrm{Mbps}$ \\
Maximum backoff stage & 5 \\
Maximum transmission attempt & 8 \\
Minimum contention widow & 31 \\
Sensing field & $(160 \mathrm{~m} \times 200 \mathrm{~m})$ \\
Nominal transmission range & $40 \mathrm{~m}$ \\
SIFS & $10 \mu \mathrm{s}$ \\
DIFS & $50 \mu \mathrm{s}$ \\
Slot time & $20 \mu \mathrm{s}$ \\
\hline
\end{tabular}

$$
T 1=T_{D I F S}+T_{a v g} \sigma+T_{\text {trans }}
$$

$\mathrm{T} 2$, however, is sampling rate dependent, which equals to the time used for sensing an interesting event in the environment and forming the sampled bytes into a corresponding packet, assuming the sensors in each node sample asynchronously. Therefore, T2 can be expressed as:

$$
T 2=\frac{N_{D A T A}}{R_{S}}
$$

So the time interval $\mathrm{T}$ in the unsaturated situation of the 802.11 protocol can be obtained by just subtracting T1 from $\mathrm{T} 2$.

$$
\begin{aligned}
T= & \frac{N_{D A T A}}{R_{S}}-T_{D I F S}-T_{a v g} \sigma-3 T_{S I F S} \\
& -\frac{N_{R T S}+N_{C T S}+N_{D A T A}+N_{A C K}}{R_{t}}
\end{aligned}
$$

The average active neighbors, $\mathrm{M}$, is dependent on the time interval $\mathrm{T}$ only in the second and fourth condition (analyzed in subsection 3.3); if we insert equation 15 into equation 8 and 12 , respectively, $M$ can be calculated. In this way, all the variables $\left(N_{\text {neighbor }}, T_{\text {avg }}, \mathrm{M}\right.$ and $\left.\mathrm{T}\right)$ which determine the performance of the collision probability are successfully described.

\section{SIMULATION}

In this subsection, with the help of the Matlab, version 7.0.1, we will examine the result of the analytical model, visualize the performance of the collision probability, study the impact of the sampling rate, present the observations of our simulation as well as show the correlations between the collision probability in the saturated and unsaturated situation of the 802.11 protocol.

To approach the real environment, we use the topology with the given nodes randomly distributed in an area with the size 160 meters by 200 meters, where one of these nodes is referred as the sink, with the endless power supply and the minimum location assumption. We assume the transmission rate is $2 \mathrm{Mbps}$ considered in the ideal channel conditions and unchanged status. During the simulation, however, we set this value by using the unit byte per microsecond in order to be in accord with the time unit of SIFS, DIFS, slot time and the length unit of packets. All other parameter values are presented in the table 1 . 


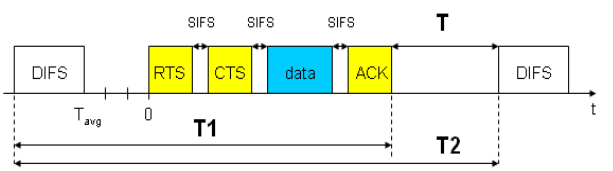

Figure 9: $\mathrm{T}$ as a function of $\mathrm{T} 1$ and $\mathrm{T} 2$

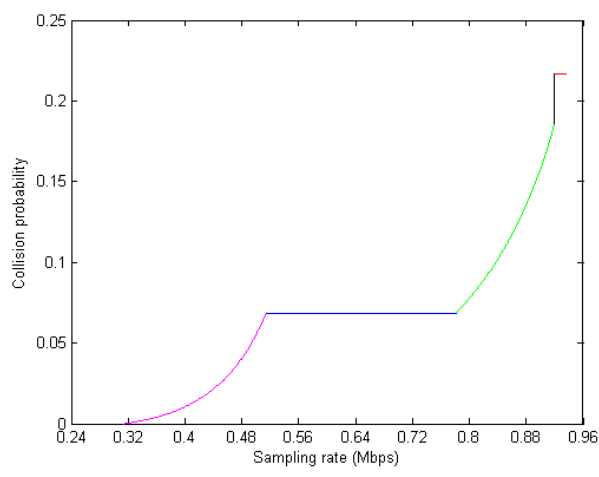

Figure 10: Collision probability in the unsaturated case

\section{DISCUSSION}

In the analytical model, the overall backoff time, the active transmitting nodes and the average neighbors are required to be integers. However, during the simulation, except for the average neighbor nodes, this requirement is very difficult to be satisfied, since some variables that cannot be simplified. These variables prevent the resolution of the equation sets if the floor integers are guaranteed. As a result, in the simulation, we could only show the asymptotic behavior of the collision probability which is drawn as curves instead of a series of straight lines.

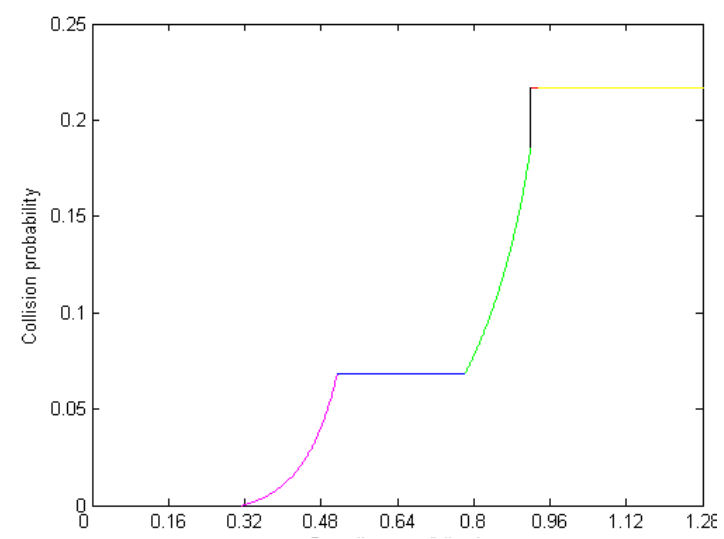

Figure 11: Overall Collision probability

As displayed in figure 10, the collision probability in the unsaturated situation of the 802.11 protocol varies from 0 to 0.22 as the sampling rate changes from 0 to $0.94 \mathrm{Mbps}$. The curve representing the collision probability starts when the sampling rate equals to $0.31 \mathrm{Mbps}$, which indicates that collision will not occur when the sensors' sampling rate is between 0 and $0.31 \mathrm{Mbps}$. In the first and third condition, since the collision probability is independent of the sampling rate, it remains a constant value that equals 0.22 and 0.68 when the sampling rate ranges between 0.92 and $0.94 \mathrm{Mbps}$ and 0.51 and $0.78 \mathrm{Mbps}$, respectively. However, in the second and fourth conditions where the sampling rate ranges between 0.78 and $0.9 \mathrm{Mbps}$; and 0 and $0.5 \mathrm{Mbps}$, the collision probability behaves as a curve instead of a straight line parallel to the rate axis.

It is worth to mention that a gap stands between the lines of the collision probability in the first and second condition, which is denoted by a black vertical beeline. This phenomenon appears due to the different formulas which evaluate the active neighbor nodes in these two conditions. As a consequence, once the sampling rate increases to $0.92 \mathrm{Mbps}$, the collision probability jumps a big step directly from 0.19 to 0.21 . In addition, the collision probability does not increase any more once the sampling rate reaches the critical value $0.94 \mathrm{Mbps}$, since it reaches the saturated situation of the 802.11 protocol, described by the yellow line in the figure 11.

\section{CONCLUSION}

In this paper, we studied the collision probability of a contention based medium access control protocol in wireless sensor networks. We demonstrated that collision depends on the sampling rate, especially when it is above $0.31 \mathrm{Mbps}$, below which, however, collision appears to be insignificant and, therefore, the collision avoidance mechanism can be removed.

\section{REFERENCES}

[1] G. Bianchi. Performance analysis of the ieee 802.11 distributed coordination function. IEEE Journal on selected areas in communications, 18(3), 2000.

[2] R. Bruno, M. Conti, and E. Gregori. Optimization of efficiency and energy consumption in p-persistent csma-based wireless lans. IEEE Transactions on Mobile Computing, 1(1):10-31, 2002.

[3] X. Chao, W. Dargie, and G. Lin. Energy model for h2s monitoring wireless sensor network. In CSE '08: Proceedings of the 2008 11th IEEE International Conference on Computational Science and Engineering, pages 402-409, Washington, DC, USA, 2008. IEEE Computer Society.

[4] W. Dargie and A. Schill. Building the senceive system. In Casemans '09: Proceedings of the 3rd ACM International Workshop on Context-Awareness for Self-Managing Systems, pages 18-24, New York, NY, USA, 2009. ACM.

[5] R. Mochaourab and W. Dargie. A fair and energy-efficient topology control protocol for wireless sensor networks. In CASEMANS'08: Proceedings of the 2nd ACM international conference on Context-awareness for self-managing systems, pages 6-15, New York, NY, USA, 2008. ACM.

[6] X. Wang, G. Min, and L. Guan. Performance modelling of ieee $802.11 \mathrm{dcf}$ using equilibrium point analysis. Int. J. Wire. Mob. Comput., 3(3):201-209, 2009.

[7] M. Zimmerling, W. Dargie, and J. M. Reason. Localized power-aware routing in linear wireless sensor networks. In CASEMANS '08: Proceedings of the 2nd ACM international conference on Context-awareness for self-managing systems, pages 24-33, New York, NY, USA, 2008. ACM. 